\title{
Twitter-Based Social Support Added to Fitbit Self-Monitoring for Decreasing Sedentary Behavior: Protocol for a Randomized Controlled Pilot Trial With Female Patients From a Women's Heart Clinic
}

Marily Oppezzo ${ }^{1}, \mathrm{RD}, \mathrm{MSc}, \mathrm{PhD}$; Jennifer Tremmel ${ }^{2}$, MD; Manisha Desai ${ }^{3}, \mathrm{PhD}$; Michael Baiocchi ${ }^{4}, \mathrm{PhD}$; Danielle Ramo $^{5,6}, \mathrm{PhD}$; Mark Cullen ${ }^{7}, \mathrm{PhD}$; Judith J Prochaska ${ }^{1}, \mathrm{PhD}$

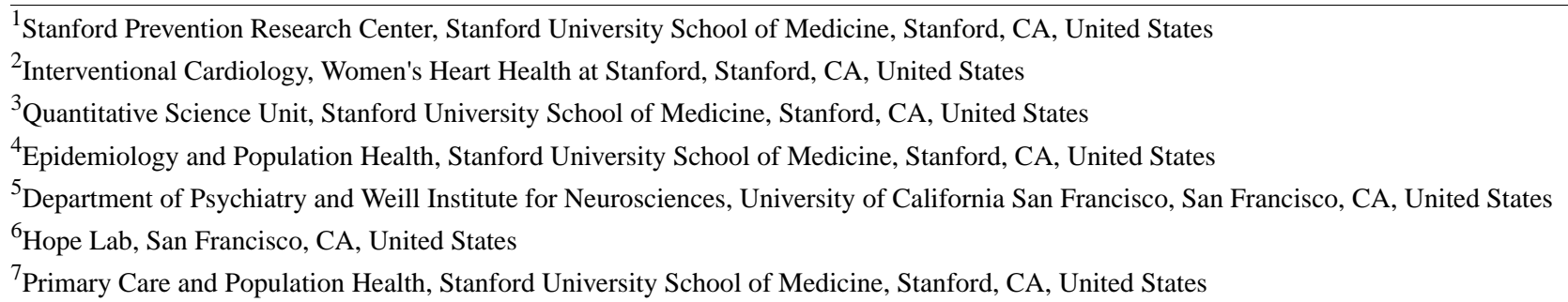

Corresponding Author:

Marily Oppezzo, RD, MSc, PhD

Stanford Prevention Research Center

Stanford University School of Medicine

Medical School Office Building

1265 Welch Road, Mail Code 5411

Stanford, CA, 94305-5411

United States

Phone: 16507246152

Email: moppezzo@stanford.edu

\section{Abstract}

Background: Prolonged sitting is an independent risk behavior for the development of chronic disease. With most interventions focusing on physical activity and exercise, there is a separate need for investigation into innovative and accessible interventions to decrease sedentary behavior throughout the day. Twitter is a social media platform with application for health communications and fostering of social support for health behavior change.

Objective: This pilot study aims to test the feasibility, acceptability, and preliminary efficacy of delivering daily behavior change strategies within private Twitter groups to foster peer-to-peer support and decrease sedentary behavior throughout the day in women. The Twitter group was combined with a Fitbit for self-monitoring activity and compared to a Fitbit-only control group.

Methods: In a 2-group design, participants were randomized to a Twitter + Fitbit treatment group or a Fitbit-only control group. Participants were recruited via the Stanford Research Repository System, screened for eligibility, and then invited to an orientation session. After providing informed consent, they were randomized. All participants received 13 weeks of tailored weekly step goals and a Fitbit. The treatment group participants, placed in a private Twitter support group, received daily automated behavior change "tweets" informed by theory and regular automated encouragement via text to communicate with the group. Fitbit data were collected daily throughout the treatment and follow-up period. Web-based surveys and accelerometer data were collected at baseline, treatment end (13 weeks), and at 8.5 weeks after the treatment.

Results: The initial study design funding was obtained from the Women's Heart Clinic and the Stanford Clayman Institute. Funding to run this pilot study was received from the National Institutes of Health's National Heart, Lung, and Blood Institute under Award Number K01HL136702. All procedures were approved by Stanford University's Institutional Review Board, \#32127 in 2018, prior to beginning data collection. Recruitment for this study was conducted in May 2019. Of the 858 people screened, 113 met the eligibility criteria, 68 came to an information session, and 45 consented to participate in this pilot study. One participant 
dropped out of the intervention, and complete follow-up data were obtained from 39 of the 45 participants ( $87 \%$ of the sample). Data were collected over 6 months from June to December 2019. Feasibility, acceptability, and preliminary efficacy results are being analyzed and will be reported in the winter of 2021.

Conclusions: This pilot study is assessing the feasibility, acceptability, and preliminary efficacy of delivering behavior change strategies in a Twitter social support group to decrease sedentary behavior in women. These findings will inform a larger evaluation. With an accessible, tailorable, and flexible platform, Twitter-delivered interventions offer potential for many treatment variations and titrations, thereby testing the effects of different behavior change strategies, peer-group makeups, and health behaviors of interest.

Trial Registration: ClinicalTrials.gov NCT02958189, https://clinicaltrials.gov/ct2/show/NCT02958189

International Registered Report Identifier (IRRID)： DERR1-10.2196/20926

(JMIR Res Protoc 2020;9(12):e20926) doi: 10.2196/20926

\section{KEYWORDS}

support group; sedentary behavior; eHealth; Twitter; Fitbit; intervention; behavior change theory; mobile phone

\section{Introduction}

\section{Background}

Sedentary behavior is a major risk factor for heart disease and early mortality, particularly among women [1-6]. Due to technological conveniences and more office-bound occupations, prolonged sitting now accounts for over half of our waking hours [7-9]. Fortunately, a growing evidence base indicates that even small increases in light-intensity physical activity can result in cardiometabolic, physical function, and mental health benefits in women and men of any age [10-12]. Compared to physical activity interventions, relatively few interventions have addressed prolonged sitting [13,14], and there is a need for more controlled trials to specifically target reductions in objectively measured prolonged sedentary behavior [14,15], especially in women at risk or with extant heart disease [1-6].

\section{Social Media Interventions}

Social media, in particular, or web apps that allow users to receive, generate, react to, and share content via a social network are a specific type of web-based platforms harnessed in health interventions, with modest effectiveness [16]. Different from a single bout of planned exercise, prolonged sitting occurs in multiple contexts throughout the day. Interventions with an accessible, dynamic web-based component like social media, available at any and many timepoints, may show a particular benefit for reducing sedentary behavior [17]. Not only can social media deliver in-context health information with broad reach, customizability, and easy access [18,19], it can also allow users to react and add to the content and provide social support to other users (an evidence-based behavior change technique [16,20-22]).

Twitter is a choice intervention platform, with high prevalence of use $(73 \%$ of the adults in the United States use social media sites, and the majority use these sites daily [23]) and often accessed via mobile devices $(80 \%$ of the Twitter users access via their mobile devices [24]). Twitter has the capability of allowing for private groups to be created that are protected from the public and even friends, making it ideal for delivering and privatizing a research intervention. Additionally, Twitter messages (called tweets) have a 280-character limit, which enables messages to be short and accessible. Often used as a supplementary aid, the potential for utilizing Twitter as a stand-alone to deliver health behavior interventions is not yet fully realized [16]. When used, engagement strongly predicts the benefits $[25,26]$. Tweet2Quit, a Twitter-based intervention for smoking cessation, is among the first successful interventions designed to promote smoking cessation with sustained long-term engagement and maintenance of changed behavior [27,28].

\section{Wearable Activity Trackers}

Consumer-based wearable activity trackers provide real-time self-monitoring feedback for the consumers on their activities throughout the day. Brickwood et al [29] found a nonsignificant decrease in sedentary behavior in their meta-analysis, but in their meta-analysis, Compernolle et al [30] found interventions specifically targeting sedentary behavior and using objective self-monitoring significantly reduced sedentary time. Fitbits are used in this study, and these devices have a feature, "active hours," which tracks the consumer's hours of 250 steps or more (equivalent to 2 minutes of walking). A secondary benefit of using a consumer-grade device is the opportunity to track the objective physical activity of participants for the entire duration of the intervention, thereby complimenting the short-duration periods measured by accelerometers [31,32].

\section{Behavior Change Techniques}

The CONSORT (Consolidated Standards of Reporting Trials) guidelines call for precise reporting of behavior change interventions [33]. Michie et al [34] provided a taxonomy of behavior change techniques, which provide consistency and comparability across interventions, as well as facilitate identification of successful components within an intervention. The participants in our study were divided into 2 groups: the control group that only used Fitbit and the treatment group that used Fitbit and Twitter engagement, in which selected behavior change techniques were delivered. Therefore, self-monitoring with Fitbit [30] was used for both groups of participants (see Multimedia Appendix 1 for the sample of messages, the accompanying behavior change techniques, and theoretical domains [35], the behavior change theories that informed all the messages [21,36,37], and our study categorization). To simplify characterization and comparison within our intervention, we organized the behavior change techniques we 
delivered via the Twitter intervention into 2 types of strategies: those that occur inside the mind, or internal strategies, and those that utilize the world outside the mind, or external strategies $[38,39]$. Internal strategies target one's cognitions about the behavior to be motivated, for example, promoting a growth mindset or focusing on the anticipated benefits of the behavior. External strategies utilize the outside world to help motivate the behavior, for example, using a timer to remind oneself to move or enlisting a friend to go for a walk. We used both types of strategies in this pilot study to target moving more often or breaking up prolonged sitting.

\section{Tweet4Wellness Intervention}

This pilot study builds upon the successful, private Twitter-based social support group intervention structure of Tweet2Quit and applies it to the less studied space of decreasing sedentary behavior, with the intervention titled as Tweet4Wellness [20]. Tweet4Wellness intervention messages are delivered daily to a private peer support group; these messages utilize behavior change techniques categorized by internal and external strategies that are shown to be effective in changing behavior and they target increased movement throughout the day $[21,34,35,37,40,41]$. Tweet4Wellness is paired with a wearable device (Fitbit) to facilitate objective self-monitoring. The aim of this intervention is to see if adding a social component (Tweet4Wellness) would be feasible, acceptable, and lead to greater reductions in sedentary behavior relative to self-monitoring (Fitbit) alone. A secondary benefit of using a consumer-grade device is the opportunity to track the objective physical activity of the participants for the entire duration of the intervention, thereby complimenting the short-duration periods measured by accelerometers [31,32].

\section{Aims of This Study}

Our primary aims are to test the feasibility, acceptability, and preliminary efficacy of Tweet4Wellness for reducing sedentary behavior when paired with self-monitoring compared to self-monitoring alone. We hypothesized that the intervention would be feasible and acceptable for women recruited from the heart clinic. The outcomes of feasibility and acceptability were operationalized by a number of emails and phone call assistance from study staff to participants; feedback from the participants informally and via a survey on usability, likability, and suggestions for improvement; and description of the study procedure challenges. Research has yet to define the clinically relevant length of a break or length of prolonged sitting that impacts health risks, and a single measure does not adequately capture all the sedentary behavior features relevant to health [42]. Therefore, to test the preliminary efficacy on sedentary behavior outcomes, we used several measures of sedentary behavior, each capturing a different component. One is an outcome provided by Fitbit: the number of active hours or daily hours achieving over 250 steps (Fitbit's estimate equivalent of 2 minutes of walking). We chose this measure because it is the trackable behavior each participant could self-monitor throughout the intervention. We also used the following interpretable measures proposed by Byrom et al [43] in their comprehensive coverage of sedentary behavior measurement: the maximum daily sedentary bout (longest, continuous, unbroken periods of sitting/no steps); daily weighted median sedentary bout (a measure of centrality capturing the distribution of the sedentary bouts); the total number of sedentary minutes; and the total number of steps (a measure of physical activity overall) [43]. We hypothesized that Tweet4Wellness + Fitbit group will increase their active hours (hours over 250 steps), increase their information entropy, have shorter maximum sedentary bouts and daily weighted median sedentary bouts, and fewer total number of sedentary minutes relative to baseline, compared to the Fitbit-only group.

The secondary aims will test the same hypotheses based on the 8.5-week follow-up period with no active intervention. The exploratory aims are within the Tweet 4 Wellness group. We will investigate the differences in the sedentary behavior summary measures by Twitter engagement or the number of tweets sent over the study period and by the type of behavior change strategy delivered each week (internal vs external).

\section{Methods}

\section{Study Design: Design, Recruitment, and Inclusion/Exclusion Criteria}

\section{Trial Design}

This pilot study was a 2-group randomized design. Treatment and control groups were run concurrently in time, and the study setting was largely virtual with an option for in-person orientation session attendance.

\section{Recruitment}

Figure 1 shows the CONSORT diagram for this study. Participants were recruited via an email from the Stanford Research Repository System to women who had been referred to or seen at the Women's Heart Health clinic (we did not require a diagnosis of heart disease). Recruitment emails were securely sent to women by the director of the Women's Heart Health clinic and they were provided information about the study with a link to the screener to confirm eligibility. 
Figure 1. CONSORT diagram. CONSORT: Consolidated Standards of Reporting Trials.

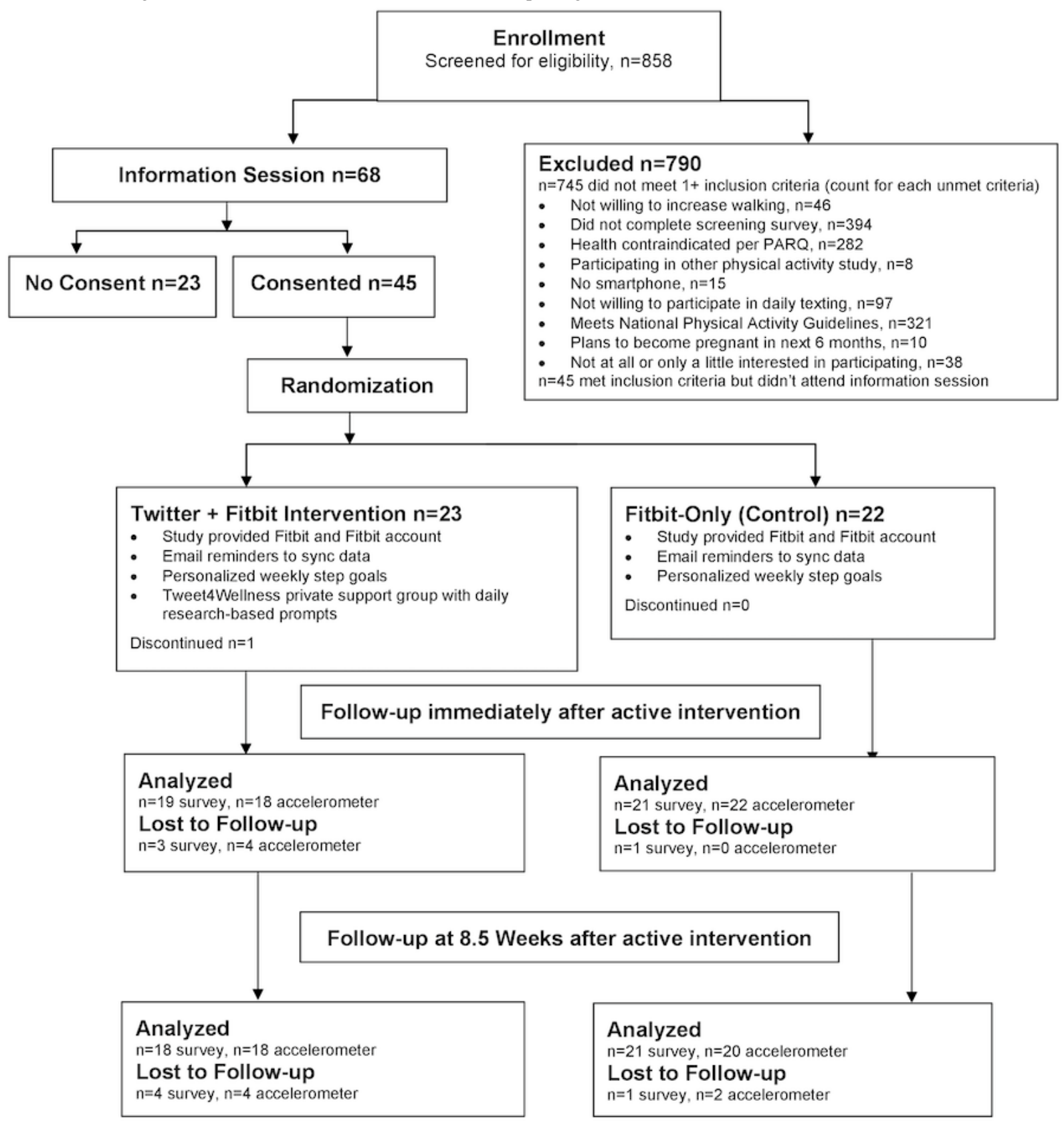

\section{Inclusion Criteria}

The major inclusion criteria on the screener were being female, older than 18 years, willing to participate in daily tweets or texts for up to 13 weeks, having an active email account to receive study communications, having a mobile phone with unlimited texting and internet to receive text and Twitter messages, being familiar with communicating on Twitter, Facebook, or other social media (proxy for computer literacy), English-speaking (for group communication), and answer No to all 7 of the Physical Activity Readiness Questionnaire questions (physical readiness to safely perform physical activity [44]).

\section{Exclusion Criteria}

The major exclusion criteria were having health or physical limitations for walking (as the study encouraged walking to break up prolonged bouts of sitting) and meeting current physical activity guidelines of 150 minutes of moderate or 75 minutes of vigorous activity per week.

\section{Procedure}

Table 1 shows the study flow from enrollment and allocation to condition (Twitter + Fitbit or Fitbit-only) through the 13-week intervention, with assessments at baseline, 13 weeks, and 21.5 weeks. 
Table 1. Study flow.

\begin{tabular}{|c|c|c|c|c|c|c|c|}
\hline \multirow[t]{3}{*}{ Study activity } & \multicolumn{7}{|c|}{ Study period and timeline } \\
\hline & Enrollment & Allocation & Postallocation & & & & \\
\hline & 0 month & 0 month & 1 month & 2 months & 3 months & 4 months & 5 months \\
\hline \multicolumn{8}{|l|}{ Enrollment } \\
\hline Eligibility screen & $\checkmark$ & & & & & & \\
\hline Information session & $\checkmark$ & & & & & & \\
\hline Informed consent & $\checkmark$ & & & & & & \\
\hline Randomization & & $\checkmark$ & & & & & \\
\hline \multicolumn{8}{|l|}{ Treatments } \\
\hline Twitter + Fitbit & & & $\checkmark$ & $\checkmark$ & $\checkmark$ & & \\
\hline Fitbit only & & & $\checkmark$ & $\checkmark$ & $\checkmark$ & & \\
\hline \multicolumn{8}{|l|}{ Measures } \\
\hline Web-based surveys & & & $\checkmark$ & & $\checkmark$ & & $\checkmark$ \\
\hline 7-day accelerometers & & & $\checkmark$ & & $\checkmark$ & & $\checkmark$ \\
\hline Continuous Fitbit monitoring & & & $\checkmark$ & $\checkmark$ & $\checkmark$ & $\checkmark$ & $\checkmark$ \\
\hline
\end{tabular}

The participants had 6 scheduled touchpoints: an orientation session (in-person or remote attendance), web-based consent and baseline survey (electronic via REDCap [Research Electronic Data Capture] [45]), a phone call with the research staff for setting up study accounts, baseline accelerometer wear (remote), posttreatment web-based survey (at 13 weeks after the baseline, electronic), accelerometer wear (mailed with a prepaid return package), and follow-up web-based survey (at 21.5 weeks after the baseline, electronic). All surveys included both closed and open-ended questions and they were emailed automatically by REDCap, with automatic reminders sent up to 2 times if participants did not respond. Participants received US \$10 gift cards for completion of the 13-week posttreatment and 21.5-week follow-up surveys, and US \$10 gift cards for returning the accelerometers at post and follow-up assessments. Figure 1 shows the CONSORT flow of the participants through the trial. All procedures were approved by Stanford University's Institutional Review Board, \#32127, and registered at clinicaltrials.gov, NCT02958189, prior to beginning data collection. This study was based on the most recent protocol version, June 2019. During the course of the study, there were no major study revisions.

\section{Orientation Session}

Eligible women per the screener were contacted from the study email and invited to attend a mandatory orientation session either in-person or in a video conference. The session described the study design, timeline, and consent; explained research methods principles; and incorporated motivational interviewing techniques. The session was based on Goldberg and Kiernan's [46] work showing increased participant retention [46,47]. One group activity had participants think through the pros and cons of being in each condition (Twitter + Fitbit or Fitbit-only) or choosing to not participate. Women who were still interested sent an email after the orientation, after which they received the electronic consent form and the baseline questionnaire. A waiver of documentation was obtained for study consent. Each page had the following sentence before moving onto the next page: "Please check here to indicate you have read and understand this information."

For this pilot study, of the 34 women who attended the web-based orientation session, 18 (53\%) consented; of the 30 women who attended the in-person session, 23 (77\%) consented; and of the 4 women who had private phone call orientation sessions, all consented. The orientation session was the only in-person meeting, while the remaining parts of the study, including data collection, were remote.

\section{Randomization}

Randomization was done in blocks with a 1:1 randomization ratio to achieve balance across the 2 conditions (Twitter + Fitbit or Fitbit-only). After 10 participants consented and completed the baseline questionnaires, they were sorted from most to least on total weekly minutes of physical activity reported on the baseline survey. The first participant was randomized to either treatment or control using a random number generator website [48], and the next participant in the pair was allotted to the other condition. The process was repeated until all of the consented participants were randomized (the last randomization in the pilot included 15 participants).

\section{Account Set-Up}

All participants scheduled a phone call with the study staff to set up their Fitbit devices and study-provided Fitbit accounts, and they downloaded the Fitbit app on their smartphones. Participants were instructed to begin wearing the Fitbit continuously for the entire study period after the baseline accelerometer week and to open their app daily to sync their data. They could reach out to study staff via email to troubleshoot problems.

Twitter + Fitbit group participants also set up their study-provided Twitter accounts and downloaded the Twitter app on their smartphones during the call. For each Twitter 
account, all privacy features were turned on to prevent participants from being searched on Twitter or from having their tweets seen by people outside the treatment group. Other group norms were provided: participants were not to follow any accounts outside the private Twitter group, they were to keep their personal Twitter accounts separate from the study account, and they were instructed to use proper etiquette when messaging (no personal attacks or bullying). Tweets were monitored daily by the study staff.

\section{Intervention}

Both the treatment (Twitter + Fitbit) and control (Fitbit-only) group received the Fitbit self-monitoring component. The treatment group also received the Twitter intervention.

\section{Fitbit Self-Monitoring Component}

All participants, both control $(n=22)$ and treatment $(n=23)$, received a study-provided Fitbit Inspire and study-provided Fitbit account connected to Fitabase, a web-based analytics and data aggregation system (Small Steps Labs). The Fitbit allowed for self-monitoring of daily steps and number of active hours. Participants were encouraged to open the Fitbit app daily to monitor their activities and to sync their data with Fitabase. When a participant did not sync their Fitbit data for over 24 hours, a study team member who monitored the Fitabase data site daily would send an email reminding the participant to open the app and sync their device. Additionally, all study participants received weekly text messages to achieve an average of $10 \%$ more steps per day, given their average step count the previous week (automatically generated and sent by the study platform). This was done to provide personalization as well as encourage data syncing (required for accurate personalized step goals). Weekly texts stopped after 13 weeks of active treatment, while Fitbit data were still collected during the follow-up period.

\section{Twitter Component}

Treatment participants were signed up with study-provided accounts for a private Twitter group. The study-provided account preserved participant anonymity, allowed for study control of the privacy settings within the group, allowed researchers to discontinue an account if any personal threats or harming messages were posted by a participant; and facilitated tweet captures if any direct messages between group members occurred. Twitter group participants received daily prompts suggesting a behavior change strategy and encouraging group sharing and discussion (see Multimedia Appendix 1 for examples). The behavior change strategies in the daily prompts were informed by theories of behavior change, namely, Bandura's Social Cognitive Theory [21], Prochaska's Transtheoretical Model of Behavior Change [49], Dweck's Implicit Theories model [50,51], and Gollwitzer's implementation intentions [37]. We also tied each strategy to a behavior change technique in the taxonomy for consistent language and constructs proposed by Michie et al [34]. All of the strategies were more broadly organized into 2 categories: (1) internal strategies, directed at thoughts or self-talk (eg, "by paying attention to how you feel before and after a walk, you can start to 'show' your brain the real-time benefits of physical activity. Each time you do this, you strengthen the connection.
Try this today for your 'move more' walk. Share how it worked!') and (2) external strategies, directed at changing the outside world to help achieve the behavior change (eg, "What's your 'slump time' of the day when you feel most rundown? Even light movement can combat it and will replace less healthful fixes (like candy!). Schedule a 5-minute walk during your slump time today and how you will remind yourself to take it. Have you tried this before?"). Having 2 categories simplified message scheduling and allowed for exploratory analyses to compare the relative effectiveness of each category (eg, did weeks with external strategies result in more sedentary behavior reduction than weeks with internal strategies?). Each week alternated between 3 sedentary behavior goals: move more (total steps per day), move more often (frequency of steps per day), and sit less (breaking up prolonged sitting). The message organization scheme is shown in Multimedia Appendix 1. Participants were encouraged to tweet in the group daily either for support or to address the prompt or both. The research staff monitored the daily activity for bullying or threatening messages.

Treatment group participants also received daily automated texts directly in their phone, providing feedback on their tweeting behavior on the prior day, praising tweeters, and encouraging nontweeters to engage. The automated text, delivered via the study web platform, considered the prior day(s)' activity. If a participant tweeted within the previous 24 hours, they received a praise or reinforcement text at the following frequencies: every other day for weeks 1-2, every 3 days for weeks 3-6, every 4 days for weeks 7-10, and every 5 days for weeks 11-13. If a participant did not tweet within the previous 24 hours, they received an encouragement or a reminder to tweet text at the following frequencies: every day for weeks 1-4, every other day for weeks 5-10, and every 3 days for weeks 11-13. The automated text message frequency was originally scheduled for every day; however, several participants complained about the frequency and 1 participant requested that the messages stop (which was honored for that participant); therefore, a gradated schedule beginning week 5 was created for all. The frequency of encouragement texts remained higher than that of the praise texts, as encouragement has been shown to improve or increase engagement [27,28].

\section{Measures}

\section{Surveys}

The survey questions assessed the participants' goals and motivations regarding walking and sedentary behavior, access to green environments, self-efficacy to make physical activity changes, and current physical activity status (see Multimedia Appendix 2 for sample questions [21,50-55]).

\section{Feasibility}

Feasibility was measured in several ways. Use of the self-monitoring component, Fitbit, was measured via Fitabase, with number of days with no steps considered as nonwear/nonsynced days. Use of the Twitter intervention was determined by the number of sent tweets and number of days that the participant tweeted. The ability to recruit was measured by the proportion of the screened eligible women/women sent 
emails and the proportion of interested eligible women/women who attended the orientation. The acceptability of the Fitbit component was assessed for both conditions via several close-ended survey questions: some questions on support (eg, I felt I received a significant amount of support for being more active throughout the day when using Fitbit, 6-point Likert scale from strongly disagree to strongly agree) and some questions on perceived utility of the added adherence features (eg, how helpful did you find the weekly step goal texted to you, 5-point Likert scale from not at all helpful to extremely helpful). Acceptability of the Twitter component was assessed via close-ended survey questions parallel to the Fitbit questions and 2 open-ended questions: "What did you find helpful/would you change about the Twitter support group?" Emails sent and received by the study staff, troubleshooting issues, and various procedural challenges were all tracked and documented.

\section{Behavioral Outcomes}

Sedentary behavior was measured in 2 ways. First, participants wore triaxial accelerometers (wrist-worn Axivity AX3, [Newcastle upon Tyne, UK] or GENEActiv [Activinsights Limited, Cambridge, UK] [56]) continuously for 7 days at baseline, posttreatment, and at 8.5 weeks follow-up. Second, after the initial baseline accelerometer data collection, participants wore Fitbits continuously throughout the 21.5 weeks of the study and follow-up. The summary measures of the sedentary behavior we derive from these devices and time periods as behavioral outcomes are averaged at the day level: the number of active hours, the maximum sedentary bout length, the weighted median sedentary bout length, total sedentary minutes, and total step count.

\section{Exploratory Outcomes}

Engagement via tweeting was measured as the number of tweets sent and the number of days the participant tweeted. Internal versus external strategies will be separated into 2 groups, with time as a factor for analyses (week 1 internal vs week 5 internal).

\section{Analysis Plan for Pilot Data}

We will describe the feasibility outcomes both quantitatively (descriptive statistics of survey responses and engagement data) and qualitatively (describing unexpected events). The mixed-effects models that will be used to analyze the change from baseline to postintervention and at follow-up on the sedentary behavior outcomes by condition are outlined in more detail in Multimedia Appendix 3. Exploratory aims looking within the Twitter condition only for differences by the type of strategy (internal vs external) will add strategy type as a predictor to the mixed-effects model and use the number of tweets and number of days tweeted as engagement covariates.

\section{Trial Sample Size/Data Safety and Privacy}

\section{Sample Size}

Given this was a pilot, we intended to run all eligible and interested participants. The Tweet2Quit results, the Twitter-based intervention that the current Tweet4Wellness was based on, and research on active web-based participation group size $[20,27,28]$ suggested a Twitter group size of 17-25.

\section{Data Safety and Privacy}

As the intervention was a low risk, a data safety monitoring board was not required. The study recorded any adverse events in the Food and Drug Administration study binder. Data were collected and kept in secure web-based databases such as REDCap [45] that are password-protected with access limited to the study team. Daily Twitter activity was monitored by the study staff.

\section{Results}

The initial study design funding was obtained from the Women's Heart Clinic and the Stanford Clayman Institute. Funding to run this pilot study was received from the National Institutes of Health's National Heart, Lung, and Blood Institute under Award Number K01HL136702. All procedures were approved by Stanford University's Institutional Review Board, \#32127 in 2018, prior to beginning data collection. Recruitment for this study was conducted in May 2019. Of the 858 people screened, 113 met the eligibility criteria, 68 came to an information session, and 45 consented to participate in this pilot study. One participant dropped out of the intervention; complete follow-up data were obtained from 39 of the 45 participants (87\% of the sample). Data were collected over 6 months from June to December 2019. Feasibility, acceptability, and preliminary efficacy results are being analyzed and will be reported in the winter of 2021.

\section{Discussion}

\section{Principal Findings}

This study intervention and design were built upon the positive findings of the Tweet2Quit smoking cessation platform $[20,27,28]$. In this study, we extend the intervention framework to sedentary behavior reduction in female patients at a women's heart clinic. We investigated the additive effects of Tweet4Wellness on top of providing a Fitbit with weekly personalized step goals for reducing sedentary behavior. We tested the feasibility, acceptability, and preliminary efficacy of the Twitter + Fitbit and Fitbit-only conditions to inform a larger trial.

\section{Strengths and Limitations}

There are several strengths to the Tweet4Wellness intervention. First, it contributes to the literature on sedentary behavior reduction, where there is a need for more randomized controlled trials that primarily focus on prolonged sitting [14]. Sedentary behavior is an independent risk factor for heart disease, particularly in women, $[2,4,6]$ and is less studied than physical activity interventions $[1,2,4,14]$. Second, with a private social media group, it allows for mutual social support with the ability to automatically deliver intervention content. Third, this intervention utilizes a consumer-grade product to track daily activity throughout the entire intervention period, which provides 2 simultaneous benefits: (1) participants can self-monitor their behavior, thereby increasing their motivation to wear the Fitbit compared to accelerometers, which provide no user feedback [30,31] and (2) it provides data on the entire time course of the trial, thereby complementing the endpoints 
where accelerometers were used. Finally, the intervention uses behavior change theories to inform daily messages, ties messages to a commonly used taxonomy of behavior change techniques to aid in cross-study comparisons, and organizes the messages into 2 categories (internal and external) to facilitate comparison of the effectiveness within our study. Given the behavioral design, condition blinding was not feasible. Another limitation is that the conditions are not balanced for attention, as the Tweet4Wellness condition had daily intervention touchpoints. A strength of the additive design of this study is that an active treatment was offered to all who were eligible, potentially increasing enrollment and retention.

With both groups wearing Fitbits, this study has the advantage of providing rich data to fill in the gaps between standard accelerometer measurement timepoints. Challenges include the numerous issues that come with free-living data collection, and operationalizing distinctions between nonwear and sedentary behavior in the Fitbit in the absence of current research consensus. Therefore, the design purposefully has participants wearing both the triaxial accelerometer and the Fitbit during posttreatment and follow-up periods to allow for cross-comparison of device outputs.
Owing to the small sample size of this pilot study, we are not powered to test full efficacy. We instead look for trends in the sedentary behavior to serve as preliminary findings to inform a full-size powered randomized controlled trial to evaluate efficacy.

\section{Conclusions}

If Tweet4Wellness is found to be feasible and acceptable and has some preliminary evidence of efficacy with regard to reduced sedentary behavior, these pilot findings would guide any adjustments in scaling to a full-size randomized controlled trial to evaluate efficacy. Tweet 4 Wellness could provide a far-reaching program for anyone to receive social support from others to reduce sedentary behavior, while also learning behavior techniques for change. Given the current shelter-in-place orders during the COVID-19 pandemic, evaluating the evidence of remote participation, health promotion platforms, and protocols is of timely value. Future studies will titrate the active ingredients in this protocol, vary the group dynamics to have mixed sex groups, and identify the optimal frequency of intervention messaging to maximize long-term engagement.

\section{Acknowledgments}

The initial study design funding was obtained from the Women's Heart Clinic and the Stanford Clayman Institute. Funding to run this pilot study was received from the National Institutes of Health's National Heart, Lung, and Blood Institute under Award Number K01HL136702. The sponsors were not involved in any of the research or analyses. The trial above would not have been completed without the dedicated help of several persons. We recognize Dr. Cornelia Pechmann at the University of California at Irvine for her seminal efforts in developing the original Tweet2Quit study, a social media-based smoking cessation intervention. Douglas Calder, with technical and intervention experience from the Tweet2Quit trials, provided technical support and account oversight for this trial. Mark Sanders and Lauren Wegner were the key research assistants aiding in participant communications, account set-ups, and data collections. A special thank you to Dr. Gotzone Garay, Brynn Kronn, and Stephanie Middleton for their detailed organization and management. We also thank Dr. Ashley Sanders-Jackson for her help with the initial analysis and refinement of the intervention messages.

\section{Authors' Contributions}

MO and JJP designed the intervention, assessments, and protocol. JJP and Dr. Cornelia Pechmann's original project Tweet2Quit was the template for this study. MO and JJP designed the intervention messages. JT aided in the recruitment, mentorship, and provided medical oversight of the participants, as well as aided the research coordinators who organized the study mailings and data collection. MD is an advisor on the grant and provided guidance with the design and data analyses. MB aided in the study design, randomization schemes, statistical instruction, and mentorship of MO. MC provided guidance and mentorship to MO to help navigate the challenges of running clinical trials. DR provided guidance on the social media aspects with regard to group size and engagement.

\section{Conflicts of Interest}

None declared.

\section{Multimedia Appendix 1}

Behavior change techniques and theoretical domains of Twitter intervention messages. [DOCX File, 56 KB-Multimedia Appendix 1]

\section{Multimedia Appendix 2}

Sample survey questions. [DOCX File, 208 KB-Multimedia Appendix 2] 


\section{Multimedia Appendix 3}

Description of the sedentary measures data.

[DOCX File, 17 KB-Multimedia Appendix 3]

\section{References}

1. Carter S, Hartman Y, Holder S, Thijssen DH, Hopkins ND. Sedentary Behavior and Cardiovascular Disease Risk: Mediating Mechanisms. Exerc Sport Sci Rev 2017 Apr;45(2):80-86. [doi: 10.1249/JES.0000000000000106] [Medline: 28118158]

2. Same RV, Feldman DI, Shah N, Martin SS, Al Rifai M, Blaha MJ, et al. Relationship Between Sedentary Behavior and Cardiovascular Risk. Curr Cardiol Rep 2016 Jan;18(1):6. [doi: 10.1007/s11886-015-0678-5] [Medline: 26699633]

3. Evenson KR, Butler EN, Rosamond WD. Prevalence of physical activity and sedentary behavior among adults with cardiovascular disease in the United States. J Cardiopulm Rehabil Prev 2014;34(6):406-419 [FREE Full text] [doi: 10.1097/HCR.0000000000000064] [Medline: 25068393]

4. Seguin R, Buchner DM, Liu J, Allison M, Manini T, Wang C, et al. Sedentary behavior and mortality in older women: the Women's Health Initiative. Am J Prev Med 2014 Feb;46(2):122-135 [FREE Full text] [doi: 10.1016/j.amepre.2013.10.021] [Medline: 24439345]

5. Lyden K, Keadle SK, Staudenmayer J, Braun B, Freedson PS. Discrete features of sedentary behavior impact cardiometabolic risk factors. Med Sci Sports Exerc 2015 May;47(5):1079-1086 [FREE Full text] [doi: 10.1249/MSS.0000000000000499] [Medline: 25202848]

6. Chomistek AK, Manson JE, Stefanick ML, Lu B, Sands-Lincoln M, Going SB, et al. Relationship of sedentary behavior and physical activity to incident cardiovascular disease: results from the Women's Health Initiative. J Am Coll Cardiol 2013 Jun 11;61(23):2346-2354 [FREE Full text] [doi: 10.1016/j.jacc.2013.03.031] [Medline: 23583242]

7. Lanningham-Foster L, Nysse LJ, Levine JA. Labor saved, calories lost: the energetic impact of domestic labor-saving devices. Obes Res 2003 Oct;11(10):1178-1181 [FREE Full text] [doi: 10.1038/oby.2003.162] [Medline: 14569042]

8. Matthews CE, Chen KY, Freedson PS, Buchowski MS, Beech BM, Pate RR, et al. Amount of time spent in sedentary behaviors in the United States, 2003-2004. Am J Epidemiol 2008 Apr 01;167(7):875-881 [FREE Full text] [doi: 10.1093/aje/kwm390] [Medline: 18303006]

9. Healy GN, Wijndaele K, Dunstan DW, Shaw JE, Salmon J, Zimmet PZ, et al. Objectively measured sedentary time, physical activity, and metabolic risk: the Australian Diabetes, Obesity and Lifestyle Study (AusDiab). Diabetes Care 2008 Feb;31(2):369-371. [doi: 10.2337/dc07-1795] [Medline: 18000181]

10. Lee I, Shiroma EJ, Kamada M, Bassett DR, Matthews CE, Buring JE. Association of Step Volume and Intensity With All-Cause Mortality in Older Women. JAMA Intern Med 2019 May 29 [FREE Full text] [doi: 10.1001/jamainternmed.2019.0899] [Medline: 31141585$]$

11. Piercy KL, Troiano RP, Ballard RM, Carlson SA, Fulton JE, Galuska DA, et al. The Physical Activity Guidelines for Americans. JAMA 2018 Nov 20;320(19):2020-2028. [doi: 10.1001/jama.2018.14854] [Medline: $\underline{30418471]}$

12. King A, Whitt-Glover MC, Marquez DX, Buman MP, Napolitano MA, Jakicic J, et al. Physical Activity Promotion. 2019;51(6):1340-1353. [doi: 10.1249/mss.0000000000001945]

13. Chau JY, der Ploeg HPV, van Uffelen JGZ, Wong J, Riphagen I, Healy GN, et al. Are workplace interventions to reduce sitting effective? A systematic review. Prev Med 2010 Nov;51(5):352-356. [doi: 10.1016/j.ypmed.2010.08.012] [Medline: 20801153]

14. Sutherland C, Kynn M, Cole R, Gray M. Interventions reducing sedentary behaviour of adults: An update of evidence. Health Education Journal 2019 Oct 03;79(3):362-374. [doi: 10.1177/0017896919878225]

15. Healy GN, Eakin EG, Lamontagne AD, Owen N, Winkler EAH, Wiesner G, et al. Reducing sitting time in office workers: short-term efficacy of a multicomponent intervention. Prev Med 2013 Jul;57(1):43-48. [doi: 10.1016/j.ypmed.2013.04.004] [Medline: 23597658]

16. Maher C, Ryan J, Kernot J, Podsiadly J, Keenihan S. Social media and applications to health behavior. Current Opinion in Psychology 2016 Jun;9:50-55. [doi: 10.1016/j.copsyc.2015.10.021]

17. Huang Y, Benford S, Blake H. Digital Interventions to Reduce Sedentary Behaviors of Office Workers: Scoping Review. J Med Internet Res 2019 Feb 07;21(2):e11079 [FREE Full text] [doi: 10.2196/11079] [Medline: 30730294]

18. King AC, Hekler EB, Grieco LA, Winter SJ, Sheats JL, Buman MP, et al. Harnessing different motivational frames via mobile phones to promote daily physical activity and reduce sedentary behavior in aging adults. PLoS One 2013;8(4):e62613 [FREE Full text] [doi: 10.1371/journal.pone.0062613] [Medline: 23638127]

19. Riley WT, Rivera DE, Atienza AA, Nilsen W, Allison SM, Mermelstein R. Health behavior models in the age of mobile interventions: are our theories up to the task? Transl Behav Med 2011 Mar;1(1):53-71 [FREE Full text] [doi: 10.1007/s13142-011-0021-7] [Medline: 21796270]

20. Pechmann C, Pan L, Delucchi K, Lakon CM, Prochaska JJ. Development of a Twitter-based intervention for smoking cessation that encourages high-quality social media interactions via automessages. J Med Internet Res 2015 Feb 23;17(2):e50 [FREE Full text] [doi: 10.2196/jmir.3772] [Medline: 25707037] 
21. Bandura A. Social cognitive theory: an agentic perspective. Annu Rev Psychol 2001;52:1-26. [doi: 10.1146/annurev.psych.52.1.1] [Medline: 11148297 ]

22. Davis R, Campbell R, Hildon Z, Hobbs L, Michie S. Theories of behaviour and behaviour change across the social and behavioural sciences: a scoping review. Health Psychol Rev 2015 Sep;9(3):323-344 [FREE Full text] [doi: 10.1080/17437199.2014.941722] [Medline: 25104107]

23. Social media use in the U.S. in 2019. Pew Research Center. URL: https://www.pewresearch.org/fact-tank/2019/04/10/ share-of-u-s-adults-using-social-media-including-facebook-is-mostly-unchanged-since-2018/ [accessed 2020-04-26]

24. Twitter by the Numbers: Stats, Demographics \& Fun Facts. URL: https://www.omnicoreagency.com/twitter-statistics/ [accessed 2020-04-26]

25. An LC, Schillo BA, Saul JE, Wendling AH, Klatt CM, Berg CJ, et al. Utilization of smoking cessation informational, interactive, and online community resources as predictors of abstinence: cohort study. J Med Internet Res 2008 Dec 20;10(5):e55 [FREE Full text] [doi: 10.2196/jmir.1018] [Medline: 19103587]

26. Cole-Lewis H, Ezeanochie N, Turgiss J. Understanding Health Behavior Technology Engagement: Pathway to Measuring Digital Behavior Change Interventions. JMIR Form Res 2019 Oct 10;3(4):e14052 [FREE Full text] [doi: 10.2196/14052] [Medline: $\underline{31603427]}$

27. Pechmann C, Delucchi K, Lakon CM, Prochaska JJ. Randomised controlled trial evaluation of Tweet2Quit: a social network quit-smoking intervention. Tob Control 2017 Mar;26(2):188-194. [doi: 10.1136/tobaccocontrol-2015-052768] [Medline: $\underline{26928205}$ ]

28. Prochaska JJ, Pechmann C, Kim R, Leonhardt JM. Twitter=quitter? An analysis of Twitter quit smoking social networks. Tob Control 2012 Jul;21(4):447-449 [FREE Full text] [doi: 10.1136/tc.2010.042507] [Medline: 21730101]

29. Brickwood K, Watson G, O'Brien J, Williams AD. Consumer-Based Wearable Activity Trackers Increase Physical Activity Participation: Systematic Review and Meta-Analysis. JMIR Mhealth Uhealth 2019 Apr 12;7(4):e11819 [FREE Full text] [doi: 10.2196/11819] [Medline: 30977740]

30. Compernolle S, DeSmet A, Poppe L, Crombez G, De Bourdeaudhuij I, Cardon G, et al. Effectiveness of interventions using self-monitoring to reduce sedentary behavior in adults: a systematic review and meta-analysis. Int J Behav Nutr Phys Act 2019 Aug 13;16(1):63 [FREE Full text] [doi: 10.1186/s12966-019-0824-3] [Medline: 31409357]

31. Cadmus-Bertram LA, Marcus BH, Patterson RE, Parker BA, Morey BL. Randomized Trial of a Fitbit-Based Physical Activity Intervention for Women. Am J Prev Med 2015 Sep;49(3):414-418 [FREE Full text] [doi:

10.1016/j.amepre.2015.01.020] [Medline: 26071863]

32. Cadmus-Bertram L, Marcus BH, Patterson RE, Parker BA, Morey BL. Use of the Fitbit to Measure Adherence to a Physical Activity Intervention Among Overweight or Obese, Postmenopausal Women: Self-Monitoring Trajectory During 16 Weeks. JMIR Mhealth Uhealth 2015 Nov 19;3(4):e96 [FREE Full text] [doi: 10.2196/mhealth.4229] [Medline: 26586418]

33. Boutron I, Moher D, Altman DG, Schulz KF, Ravaud P, CONSORT Group. Extending the CONSORT statement to randomized trials of nonpharmacologic treatment: explanation and elaboration. Ann Intern Med 2008 Feb 19;148(4):295-309. [doi: 10.7326/0003-4819-148-4-200802190-00008] [Medline: 18283207]

34. Michie S, Richardson M, Johnston M, Abraham C, Francis J, Hardeman W, et al. The behavior change technique taxonomy (v1) of 93 hierarchically clustered techniques: building an international consensus for the reporting of behavior change interventions. Ann Behav Med 2013 Aug;46(1):81-95. [doi: 10.1007/s12160-013-9486-6] [Medline: 23512568]

35. Cane J, O'Connor D, Michie S. Validation of the theoretical domains framework for use in behaviour change and implementation research. Implement Sci 2012 Apr 24;7:37 [FREE Full text] [doi: 10.1186/1748-5908-7-37] [Medline: 22530986]

36. Prochaska J, Velicer W. The transtheoretical model of health behavior change. Am J Health Promot 1997;12(1):38-48. [doi: 10.4278/0890-1171-12.1.38] [Medline: 10170434]

37. Gollwitzer P. Implementation intentions: Strong effects of simple plans. American Psychologist 1999;54(7):493-503. [doi: 10.1037/0003-066X.54.7.493]

38. Oppezzo M, Schwartz D. A behavior change perspective on self-regulated learning with teachable agents. In: Azevedo R, Aleven V, editors. International Handbook of Metacognition and Learning Technologies. New York, NY: Springer; $2013: 500$.

39. Duckworth AL, Gendler TS, Gross JJ. Situational Strategies for Self-Control. Perspect Psychol Sci 2016 Jan;11(1):35-55 [FREE Full text] [doi: 10.1177/1745691615623247] [Medline: 26817725]

40. Baer RA. Mindfulness Training as a Clinical Intervention: A Conceptual and Empirical Review. Clinical Psychology: Science and Practice 2006 May 11;10(2):125-143. [doi: 10.1093/clipsy.bpg015]

41. Kiernan M, Moore SD, Schoffman DE, Lee K, King AC, Taylor CB, et al. Social support for healthy behaviors: scale psychometrics and prediction of weight loss among women in a behavioral program. Obesity (Silver Spring) 2012 Apr;20(4):756-764 [FREE Full text] [doi: 10.1038/oby.2011.293] [Medline: 21996661]

42. Byrom B, Rowe DA. Measuring free-living physical activity in COPD patients: Deriving methodology standards for clinical trials through a review of research studies. Contemp Clin Trials 2016 Mar;47:172-184 [FREE Full text] [doi: 10.1016/j.cct.2016.01.006] [Medline: 26806669]

43. Byrom B, Stratton G, Mc Carthy M, Muehlhausen W. Objective measurement of sedentary behaviour using accelerometers. Int J Obes (Lond) 2016 Nov;40(11):1809-1812 [ㅌRE Full text] [doi: 10.1038/ijo.2016.136] [Medline: 27478922] 
44. Warburton D, Jamnik V, Bredin S, Gledhill N. The Physical Activity Readiness Questionnaire for Everyone (PAR-Q+) and Electronic Physical Activity Readiness Medical Examination (ePARmed-X+). 2011;4(2):3-17. [doi: 10.14288/hfjc.v4i2.103]

45. Harris PA, Taylor R, Thielke R, Payne J, Gonzalez N, Conde JG. Research electronic data capture (REDCap)--a metadata-driven methodology and workflow process for providing translational research informatics support. J Biomed Inform 2009 Apr;42(2):377-381 [FREE Full text] [doi: 10.1016/j.jbi.2008.08.010] [Medline: 18929686]

46. Goldberg JH, Kiernan M. Innovative techniques to address retention in a behavioral weight-loss trial. Health Educ Res 2005 Aug;20(4):439-447. [doi: 10.1093/her/cyg139] [Medline: 15598664]

47. Mayhew M, Leo MC, Vollmer WM, DeBar LL, Kiernan M. Interactive group-based orientation sessions: A method to improve adherence and retention in pragmatic clinical trials. Contemp Clin Trials Commun 2020 Mar;17:100527. [doi: 10.1016/j.conctc.2020.100527] [Medline: 32083219]

48. Random.org. URL: random.org [accessed 2019-06-01]

49. Prochaska JO, Velicer WF. The transtheoretical model of health behavior change. Am J Health Promot 1997;12(1):38-48. [doi: 10.4278/0890-1171-12.1.38] [Medline: 10170434]

50. Dweck C, Chiu C, Hong Y. Implicit Theories and Their Role in Judgments and Reactions: A Word From Two Perspectives. Psychological Inquiry 2009 Nov 19;6(4):267-285. [doi: 10.1207/s15327965pli0604_1]

51. Dweck C. Mindset: the new psychology of success. Choice Reviews Online 2006 Dec 01;44(04):44-2397-44-2397. [doi: 10.5860/choice.44-2397]

52. Rosenberg DE, Norman GJ, Wagner N, Patrick K, Calfas KJ, Sallis JF. Reliability and validity of the Sedentary Behavior Questionnaire (SBQ) for adults. J Phys Act Health 2010 Nov;7(6):697-705. [doi: 10.1123/jpah.7.6.697] [Medline: 21088299]

53. Lee PH, Macfarlane DJ, Lam TH, Stewart SM. Validity of the International Physical Activity Questionnaire Short Form (IPAQ-SF): a systematic review. Int J Behav Nutr Phys Act 2011 Oct 21;8:115 [FREE Full text] [doi:

10.1186/1479-5868-8-115] [Medline: 22018588]

54. Segar ML, Eccles JS, Peck SC, Richardson CR. Midlife Women's Physical Activity Goals: Sociocultural Influences and Effects on Behavioral Regulation. Sex Roles 2007 Oct 4;57(11-12):837-849. [doi: 10.1007/s11199-007-9322-1]

55. Segar ML, Eccles JS, Richardson CR. Rebranding exercise: closing the gap between values and behavior. Int J Behav Nutr Phys Act 2011 Aug 31;8:94 [FREE Full text] [doi: 10.1186/1479-5868-8-94] [Medline: 21884579]

56. Pavey TG, Gomersall SR, Clark BK, Brown WJ. The validity of the GENEActiv wrist-worn accelerometer for measuring adult sedentary time in free living. J Sci Med Sport 2016 May;19(5):395-399. [doi: 10.1016/j.jsams.2015.04.007] [Medline: 25956687]

\section{Abbreviations \\ CONSORT: Consolidated Standards of Reporting Trials \\ REDCap: Research Electronic Data Capture}

Edited by G Eysenbach; submitted 01.06.20; peer-reviewed by E Sezgin, T Ratz; comments to author 22.07.20; revised version received
11.08.20; accepted 21.08.20; published 04.12.20
Please cite as:
Oppezzo M, Tremmel J, Desai M, Baiocchi M, Ramo D, Cullen M, Prochaska JJ
Twitter-Based Social Support Added to Fitbit Self-Monitoring for Decreasing Sedentary Behavior: Protocol for a Randomized
Controlled Pilot Trial With Female Patients From a Women's Heart Clinic
JMIR Res Protoc 2020;9(12):e20926
URL: $\underline{\text { https://www.researchprotocols.org/2020/12/e20926 }}$
doi: $\underline{10.2196 / 20926}$
PMID: $\underline{33275104}$

CMarily Oppezzo, Jennifer Tremmel, Manisha Desai, Michael Baiocchi, Danielle Ramo, Mark Cullen, Judith J Prochaska. Originally published in JMIR Research Protocols (http://www.researchprotocols.org), 04.12.2020. This is an open-access article distributed under the terms of the Creative Commons Attribution License (https://creativecommons.org/licenses/by/4.0/), which permits unrestricted use, distribution, and reproduction in any medium, provided the original work, first published in JMIR Research Protocols, is properly cited. The complete bibliographic information, a link to the original publication on http://www.researchprotocols.org, as well as this copyright and license information must be included. 